Check for updates

Cite this: Chem. Sci., 2018, 9, 6981

๑ All publication charges for this article have been paid for by the Royal Society of Chemistry

\section{A simple, label-free, electrochemical DNA parity generator/checker for error detection during data transmission based on "aptamer-nanoclaw"- modulated protein steric hindrance $\uparrow$}

\author{
Daoqing Fan, ${ }^{\text {ab }}$ Yongchao Fan, ${ }^{\text {ab }}$ Erkang Wang (D) ab and Shaojun Dong (D) *ab
}

Versatile DNA logic devices have exhibited magical power in molecular-level computing and data processing. During any type of data transmission, the appearance of erroneous bits (which have severe impacts on normal computing) is unavoidable. Luckily, the erroneous bits can be detected via placing a parity generator $(\mathrm{pG})$ at the sending module and a parity checker $(\mathrm{pC})$ at the receiving module. However, all current DNA pG/pC systems use optical signals as outputs. In comparison, sensitive, facilely operated, electric-powered electrochemical outputs possess inherent advantages in terms of potential practicability and future integration with semiconductor transistors. Herein, taking an even pG/pC as a model device, we construct the first electrochemical DNA pG/pC system so far. Innovatively, a thrombin aptamer is integrated into the input-strand and it functions as a "nanoclaw" to selectively capture thrombin; the electrochemical impedance changes induced by the "nanoclaw/thrombin" complex are used as label-free outputs. Notably, this system is simple and can be operated within $2 \mathrm{~h}$, which is comparable with previous fluorescent ones, but avoids the high-cost labeled-fluorophore and tedious nanoquencher. Moreover, taking non-interfering poly-T strands as additional inputs, a cascade logic circuit (OR-2 to 1 encoder) and a parity checker that could distinguish even/odd numbers from natural numbers ( 0 to 9 ) is also achieved based on the same system. This work not only opens up inspiring horizons for the design of novel electrochemical functional devices and complicated logic circuits, but also lays a solid foundation for potential logic-programmed target detection.
Received 6th June 2018 Accepted 15th July 2018 DOI: $10.1039 / c 8 s c 02482 k$

rsc.li/chemical-science integration with semiconductor transistors, while current electrochemical logic devices are confined to basic logic gates, keypad locks, encoders/decoders, and multi-input/-output circuits, ${ }^{21-23}$ which could not meet pluralistic logical requirements. Thus, exploring multifunctional electrochemical logic systems that could not only perform novel/specific logic functions, but also engineer various logic circuits is one of the brightest subjects.

During any kind of data transmission, the occurrence of erroneous bits (which have severe impacts on normal logic computing) is an unavoidable problem. ${ }^{24-27}$ Luckily, these bits can be detected via placing a parity generator (pG) at the sending module and a parity checker (pC) at the receiving module. As described in the $\mathrm{pG} / \mathrm{pC}$ operating principles, ${ }^{\mathbf{2 4 - 2 9}}$ an even pG could generate an additional parity bit (P) and add it to the initial binary bits Dn, changing the number of 1 's $(\Sigma)$ in the DnP string to even. For example, if two bits of data (D1 and D2) are to be sent, the 2-bit even $\mathrm{pG}$ will endow a binary value to $\mathrm{P}$ (the output of $\mathrm{pG}$ ) in light of the truth table of an XOR gate (see Scheme 1A and Fig. 1A). Afterwards, the D1D2P string generated by the 2-bit even $\mathrm{pG}$ will be sent to and checked by the 3-bit even pC (Scheme 1A). If the transmission is normal, the three bits
${ }^{a}$ State Key Laboratory of Electro Analytical Chemistry, Changchun Institute of Applied Chemistry, Chinese Academy of Sciences, Changchun, Jilin, 130022, China. E-mail: dongsj@ciac.ac.cn

${ }^{b}$ University of Chinese Academy of Sciences, Beijing, 100039, China

$\dagger$ Electronic supplementary information (ESI) available: Table S1 and Fig. S1-S7. See DOI: 10.1039/c8sc02482k 


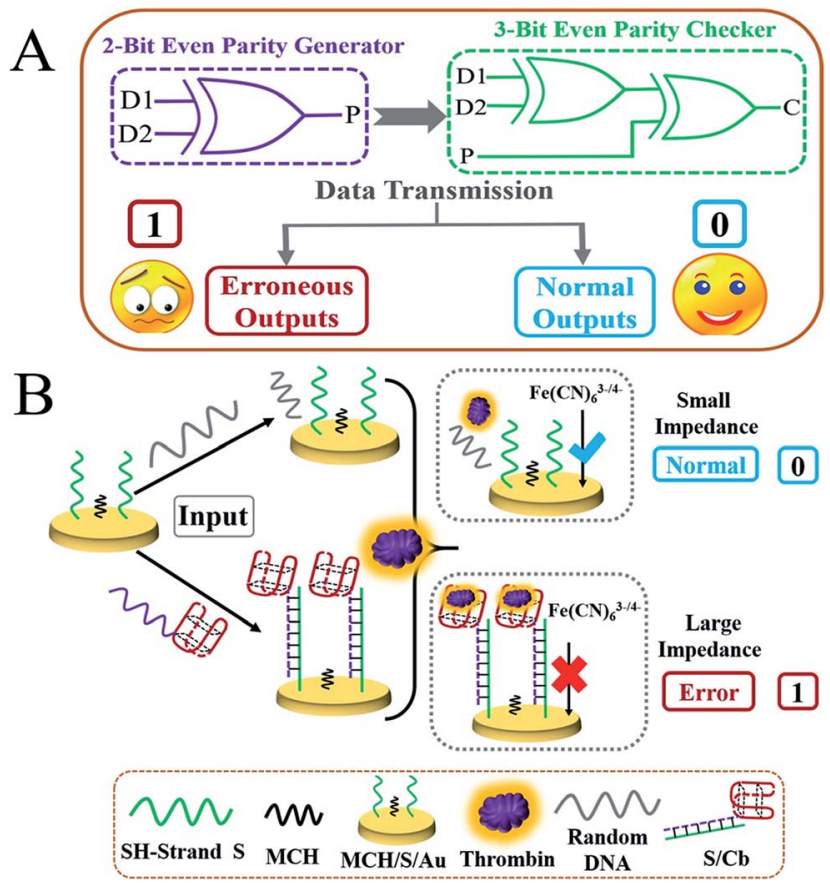

Scheme 1 (A) Equivalent logic circuit of the 2-bit even pG and 3-bit even $\mathrm{pC}$; the sad and smiling faces represent erroneous and normal outputs, respectively. (B) Schematic illustration of the operating principle of the electrochemical $\mathrm{pG} / \mathrm{pC}$ system; the red basket indicates the thrombin aptamer that functions as a "nanoclaw". The blue " $\sqrt{ }$ " and red " $x$ " symbols represent favourable and blocked electron transfer, respectively.

(D1, D2 and P) will not change. The $\Sigma$ value of the D1D2P string received by the $\mathrm{pC}$ remains even, and the $\mathrm{pC}$ exhibits a "normal" signal (Scheme 1A, the smiling face) via yielding output $C=0$. However, if errors appear during the transmission of the three bits, the $\Sigma$ value of the received wrong 3-bit string will change to odd (see Table 1), then the $\mathrm{pC}$ will give an "alarm" signal (Scheme $1 \mathrm{~A}$, the sad face) via producing output $C=1 .{ }^{24-29}$ All of the above describes the "error detection" procedure of the even $\mathrm{pG} / \mathrm{pC}$, and the odd $\mathrm{pG} / \mathrm{pC}$ qualifies similar functions. Although scientists have made great efforts to advance the $\mathrm{pG} / \mathrm{pCs}$, most of the current $\mathrm{pG} / \mathrm{pCs}$ are constructed upon semiconductor substrates, ${ }^{24-29}$ and only a few molecular pG/pCs have been achieved based on organic molecules or DNAs using optical (fluorescent or colorimetric) signals as outputs. ${ }^{28,29}$ To the best of our knowledge, no electrochemical DNA pG/pC system has ever been reported and further endeavours are required to realize it.

Electrochemical impedance spectroscopy (EIS) is widely applied to characterize the modification procedures of electrodes. $^{30}$ The semicircle region appearing at high frequencies reflects the limited charge-transfer process, and the interfacial charge-transfer resistance $\left(R_{\mathrm{ct}}\right)$ can be estimated according to the diameter of the semicircle. Actually, the EIS signals possess the inherent merits of facile testing, low cost, high sensitivity, and reliable reproducibility and have been used to probe various electrochemical biosensors. ${ }^{31-33}$ In contrast, as far as we know, DNA logic systems that are signalled by EIS signals have rarely been reported. ${ }^{34}$ According to previous reports, single-
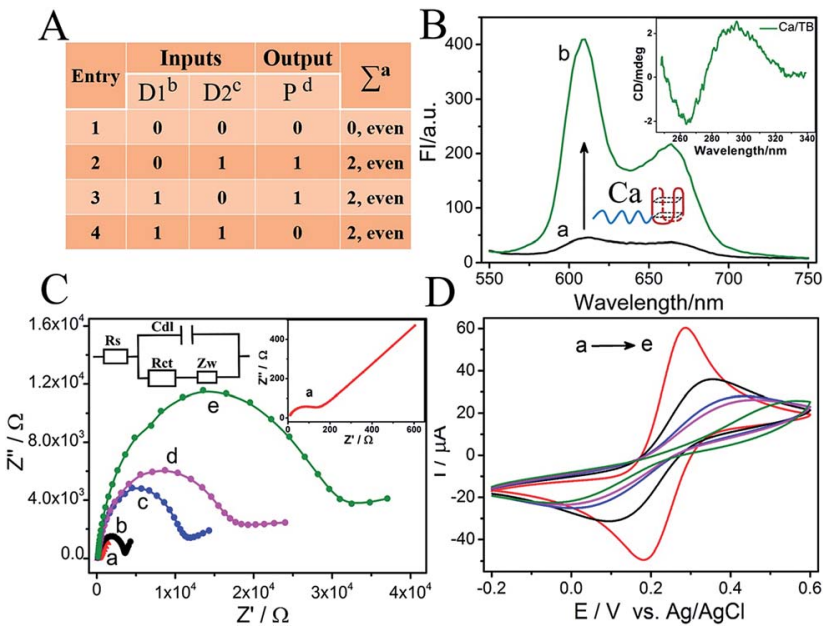

Fig. 1 (A) Truth table of the 2-bit parity generator (' ${ }^{2}$ umber of 1's in the $\mathrm{D} 1 \mathrm{D} 2 \mathrm{P}$ string, ${ }^{\mathrm{b}}$ strand $\mathrm{Ca},{ }^{\mathrm{c}}$ strand $\mathrm{Cb},{ }^{\mathrm{d}} \Delta R_{\mathrm{ct}}$ values); (B) fluorescence spectra of NMM in the absence (a) and presence (b) of Ca strand $/ \mathrm{K}^{+}$, the inset shows the $C D$ spectrum of the $\mathrm{Ca}$ /thrombin mixture; (C) electrochemical impedance spectra (Nyquist curves) of (a) a bare $\mathrm{Au}$ electrode (inset), (b) $\mathrm{S} / \mathrm{Au}$, (c) $\mathrm{MCH} / \mathrm{S} / \mathrm{Au}$, (d) $\mathrm{TB} / \mathrm{random} / \mathrm{MCH} / \mathrm{S} / \mathrm{Au}$ and (e) $\mathrm{TB} / \mathrm{Cb} / \mathrm{MCH} / \mathrm{S} / \mathrm{Au}$; and (D) cyclic voltammetry curves of the different electrodes in (C). The CV $(-0.2$ to $0.6 \mathrm{~V})$ and EIS $(0.1 \mathrm{~Hz}$ to 100 $\mathrm{kHz})$ curves were collected in $5 \mathrm{mM}\left[\mathrm{Fe}(\mathrm{CN})_{6}{ }^{3-14-}\right]$ and $0.1 \mathrm{M} \mathrm{KCl}$ aqueous solution. The scan rate for $\mathrm{CV}$ was $0.1 \mathrm{~V} \mathrm{~s}^{-1}$ and the amplitude for EIS was $5 \mathrm{mV}$.

strand DNA and other secondary structures present different repelling abilities towards the EIS probe $\left[\mathrm{Fe}(\mathrm{CN})_{6}{ }^{3-/ 4-}\right]$. And the flexibility/specificity of an aptamer to bind with macromolecules (such as protein) also enables "target-induced steric hindrance" ${ }^{35}$ to be an efficient output signal to operate electrochemical DNA logic devices.

Herein, taking an even $\mathrm{pG} / \mathrm{pC}$ as model device, we construct the first electrochemical DNA pG/pC system so far for error detection during data transmission. Innovatively, a thrombin aptamer (TBA15) is integrated into the input-strand and it functions as the "nanoclaw" to selectively capture thrombin (TB); the electrochemical impedance changes induced by the "nanoclaw/TB" complex are used as label-free outputs. Notably, this system is simple and can be operated within $2 \mathrm{~h}$, which is

Table 1 Truth table of the 3-bit even pC ( ${ }^{a}$ number of 1's in the D1D2P string, for $\mathrm{P}=1$ : ${ }^{\mathrm{b}}$ strand $\mathrm{TCb},{ }^{\mathrm{c}}$ strand $\mathrm{Cb},{ }^{\mathrm{d}}$ strand $\mathrm{Ca},{ }^{\mathrm{e}} \Delta R_{\mathrm{ct}}$ values of $\mathrm{Au}$ electrodes)

\begin{tabular}{|c|c|c|c|c|c|c|}
\hline \multirow[b]{2}{*}{ Entry } & \multicolumn{3}{|c|}{ Inputs } & \multirow{2}{*}{$\frac{\text { Output }}{\mathrm{C}^{\mathrm{e}}}$} & \multirow[b]{2}{*}{$\sum^{a}$} & \multirow[b]{2}{*}{ States } \\
\hline & $\mathrm{D} 1^{\mathrm{b}}$ & $\mathrm{D} 2^{\mathrm{c}}$ & $\mathrm{P}^{\mathrm{d}}$ & & & \\
\hline 1 & 0 & 0 & 0 & 0 & 0 , even & Ok \\
\hline 2 & 0 & 1 & 0 & 1 & 1, odd & Error \\
\hline 3 & 1 & 0 & 0 & 1 & 1 , odd & \\
\hline 4 & 1 & 1 & 0 & 0 & 2 , even & Ok \\
\hline 5 & 0 & 0 & 1 & 1 & 1 , odd & Error \\
\hline 6 & 0 & 1 & 1 & 0 & 2 , even & Ok \\
\hline 7 & 1 & 0 & 1 & 0 & 2 , even & \\
\hline 8 & 1 & 1 & 1 & 1 & 3 , odd & Error \\
\hline
\end{tabular}


comparable with previous fluorescent ones, but avoids the expensive labeled-fluorophore and tedious nanoquencher. Moreover, taking non-interfering poly-T strands as additional inputs, a cascade logic circuit (OR-2 to 1 encoder) and a parity checker that could distinguish even/odd numbers from natural numbers (0 to 9) are also achieved based on the same system.

\section{Results and discussion}

\section{Operating principle of the electrochemical pG/pC system}

As demonstrated above, the XOR gate is the basis of the $\mathrm{pG} / \mathrm{pC}$ system $^{28,29}$ and the presence of the TB aptamer (TBA15, the "nanoclaw") ${ }^{36,37}$ to capture thrombin efficiently is the key to obtaining a sufficiently high signal-to-noise $(\mathrm{S} / \mathrm{N})$ ratio. Thus, verification experiments were explored initially before the operation of the $\mathrm{pG} / \mathrm{pC}$. As shown in Scheme 1B, a Au electrode treated with SH-DNA (green strand) and blocked by $\mathrm{MCH}(\mathrm{MCH} /$ $\mathrm{S} / \mathrm{Au}$ ) was taken as the universal platform for the $\mathrm{pG} / \mathrm{pC}$. The dramatically enhanced fluorescence of $\mathrm{NMM}^{28}$ and unique $\mathrm{CD}$ peaks (negative peak at around $260 \mathrm{~nm}$ and a positive one at around $290 \mathrm{~nm}$, Fig. 1B) implied the effective binding between the nanoclaw and $\mathrm{TB},{ }^{36}$ providing an ideal secondary structure for adequate EIS changes. Before modification, the bare $\mathrm{Au}$ electrode exhibited a small $R_{\mathrm{ct}}$ and peak-to-peak difference and large peak current (Fig. 1C(a) and D(a)). After modification with SH-DNA and blocking by $\mathrm{MCH}$, the $R_{\text {ct }}$ and peak-to-peak difference of the $\mathrm{MCH} / \mathrm{S} / \mathrm{Au}$ electrode increased gradually accompanied by a decrease of peak current due to the electrostatic repulsion of negatively charged SH-DNA towards the $\left[\mathrm{Fe}(\mathrm{CN})_{6}{ }^{3-/ 4-}\right]$ probe, Fig. $1 \mathrm{C}(\mathrm{c})$ and $\mathrm{D}(\mathrm{c})$, indicating the successful fabrication of the platform.

Furthermore, the importance of the input strands and "nanoclaw" was evaluated. In the presence of random strand $\mathrm{R}$ (grey strand) without the nanoclaw (Scheme 1B), it could neither hybridize the SH-strand nor capture TB; the $R_{\text {ct }}$ presented only slight changes as for the weak nonspecific binding (Fig. 1C(d)). In contrast, in the presence of strand $\mathrm{Cb}$ (purple strand) that was integrated with the nanoclaw (the red basket), it could hybridize with the SH-strand and the nanoclaw will capture TB to form the "nanoclaw/TB" complex, leading to a significantly enhanced $R_{\mathrm{ct}}$ (Fig. 1C(e)). The peak-to-peak difference and peak current (Fig. 1D) were in good accordance with the corresponding EIS changes. The sufficiently high $\mathrm{S} / \mathrm{N}$ ratio appearing in the EIS and $\mathrm{CV}$ curves proved not only the progressive modification of the $\mathrm{Au}$ electrode, but also the feasibility of $R_{\mathrm{ct}}$ to be the output of the electrochemical DNA pG/pC system. More accurately, the $R_{\mathrm{ct}}$ changes $\left(\Delta R_{\mathrm{ct}}\right)$ were used as the outputs and the threshold value was set as $12 \mathrm{k} \Omega$ to distinguish the positive and negative outputs. $\Delta R_{\text {ct }}=R_{\text {ct }}(\mathrm{a})-R_{\text {ct }}$ (i), where $R_{\text {ct }}$ (i) and $R_{\text {ct }}$ (a) represent the $R_{\text {ct }}$ of the initial platform and that after adding inputs/TB, respectively.

\section{The 2-bit parity generator}

As illustrated in our previous work, ${ }^{28,29}$ the $\mathrm{pG} / \mathrm{pC}$ system for "error detection" during data transmission consists of an $n$-bit pG and an $(n+1)$-bit pC. Herein, a 2-bit even pG/3-bit even pC was targeted as the model and the 2-bit even pG was constructed at first according to the principle of the XOR gate (truth table, Fig. 1A). The detailed hybridization of different strands is presented in Fig. S1-S3. $\uparrow$ The $\Delta R_{\mathrm{ct}}$ value was defined as output P. As depicted in Scheme 2, strands $\mathrm{Ca}$ (blue strand) and $\mathrm{Cb}$ (purple strand) that was integrated with the "nanoclaw" and could partially hybridize with different parts of the $\mathrm{SH}$-strand acted as inputs D1 and D2, respectively. In the absence of any input (entry 1 of Scheme 2), TB could not be captured and immobilized on the surface of the electrode tightly. The final electrode exhibits weak repelling ability towards $\left[\mathrm{Fe}(\mathrm{CN})_{6}{ }^{3-/ 4-}\right]$, producing output $\mathrm{P}=0$. In the presence of any input, $\mathrm{Ca}$ or $\mathrm{Cb}$ will partially hybridize with $\mathrm{S}$ and form duplex $\mathrm{S} / \mathrm{Ca}$ or $\mathrm{S} / \mathrm{Cb}$ (entries 2 and 3 of Scheme 2); the "nanoclaw" at the end of input strands could capture TB effectively and enhance the steric hindrance evidently, yielding output $\mathrm{P}=1$ (Fig. S4†). However, in the presence of both input strands, the pre-reaction of $\mathrm{Ca}$ and $\mathrm{Cb}$ will form more stable duplex $\mathrm{Ca} / \mathrm{Cb}$ than $\mathrm{S} / \mathrm{Ca}$ or $\mathrm{S} /$ $\mathrm{Cb}^{29}$ The generated duplexes can hardly hybridize with $\mathrm{S}$ anymore and most of them will be scoured off during the washing step. And only an slightly increased $R_{\mathrm{ct}}(\mathrm{P}=0)$ was obtained after adding TB. Fig. 2A illustrates the detailed EIS curves under different input combinations. The input and output states can be further identified by the $\Delta R_{\mathrm{ct}}$ column bars (Fig. 2B), which matched the truth table (Fig. 1A) well, indicating the appropriate operation of the 2-bit even pG. The PAGE verification of strand reactions is shown in Fig. S5. $\dagger$ The corresponding optimization of the concentrations of TB and input strands is shown in Fig. S6 and S7, $\uparrow$ and the DNA sequences used in this work are given in Table S1. $\dagger$

\section{The 3-bit parity checker}

After constructing the 2-bit even pG, a 3-bit even pC was further constructed to achieve the "error detection" function. The platform of the $\mathrm{pC}$ was still the $\mathrm{MCH} / \mathrm{S} / \mathrm{Au}$ electrode, to which

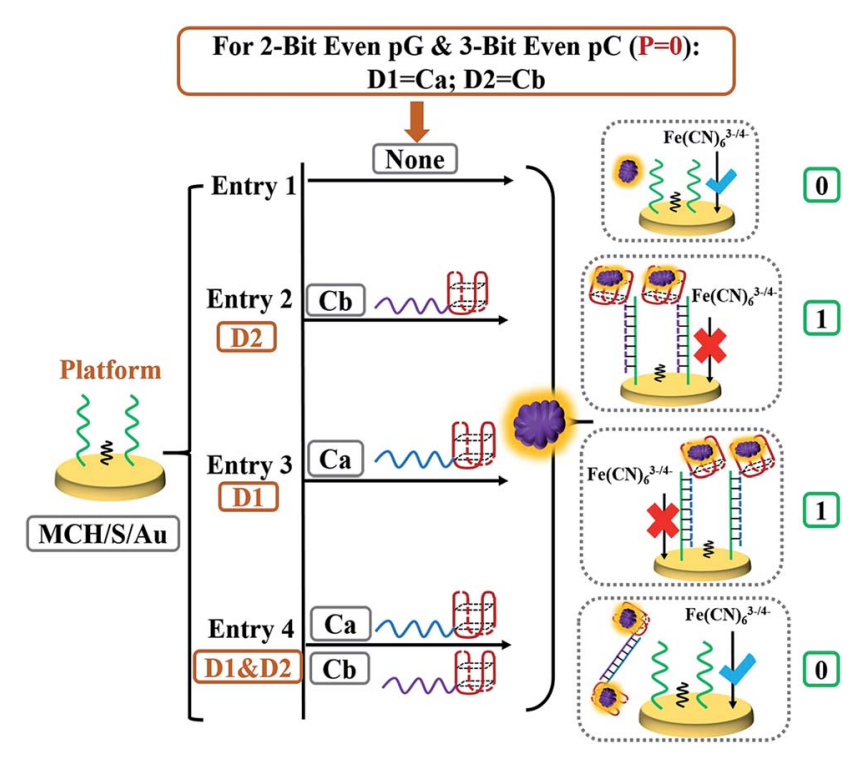

Scheme 2 Detailed DNA hybridization of different entries for the 2-bit even $\mathrm{pG}$ and 3 -bit even $\mathrm{pC}$ ( $\mathrm{P}=0$ states). Strands $\mathrm{Ca}$ and $\mathrm{Cb}$ act as inputs D1 and D2, respectively. 
A

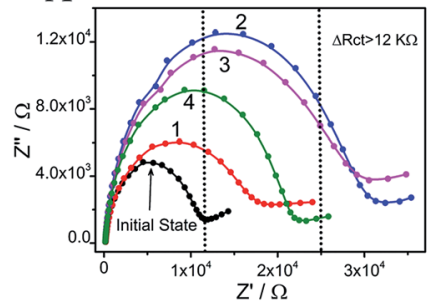

B

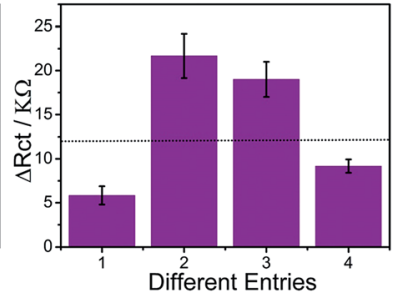

Fig. 2 (A) Electrochemical impedance spectra of the $\mathrm{MCH} / \mathrm{S} / \mathrm{Au}$ electrode under different entries: (1) TB/MCH/S/Au, (2) TB/Cb/MCH/S/ $\mathrm{Au}$, (3) TB/Ca/MCH/S/Au, and (4) TB/(Ca $+\mathrm{Cb}) / \mathrm{MCH} / \mathrm{S} / \mathrm{Au}$. (B) The $\Delta R_{\mathrm{ct}}$ column bars of the different entries.

the third input $\mathrm{P}$ was brought to meet the requirements. The input variations of the 3-bit even $\mathrm{pC}$ that are demonstrated in Table 1 can be separated into two parts..$^{\mathbf{2 4 , 2 8 , 2 9}}$ For the first part (P $=0$ states, entries 1 to 4$)$, the outputs of the pC $\left(\Delta R_{\mathrm{ct}}\right.$ was defined as output $\mathrm{C}$ now) were just the same as those of the above-constructed 2-bit even pG. The logic circuit can be considered as an XOR gate, in which the DNA reactions were analogous to the 2-bit pG and have been illustrated above. For the second part ( $\mathrm{P}=1$ states, entries 5 to 8$)$, input $\mathrm{P}$ actually executes the XNOR function that is complementary to both inputs (D1 and D2) to change the number of 1's ( $\left.\Sigma^{\mathrm{a}}\right)$ in the DnP string to even..$^{\mathbf{2 4 , 2 8 , 2 9}}$ The detailed DNA hybridizations of various entries (entries 5 to 8) are shown in Scheme 3. Herein, the third strand $\mathrm{TCb}$, that was 5 thymine units (orange part) longer than $\mathrm{Cb}$ at the $3^{\prime}$ end, was introduced as another input to achieve the function of the 3 -bit $\mathrm{pC}(\mathrm{P}=1$ states). Similar to previous reports, ${ }^{24,28,29}$ the inputs $\mathrm{Ca}, \mathrm{Cb}$ and $\mathrm{TCb}$ were redefined as follows to execute the 3-bit even $\mathrm{pC}(\mathrm{P}=1$ states) properly, $\mathrm{Ca}$ acted as input $\mathrm{P}$, while strand $\mathrm{TCb}$ and $\mathrm{Cb}$ functioned as inputs D1 and D2, respectively.

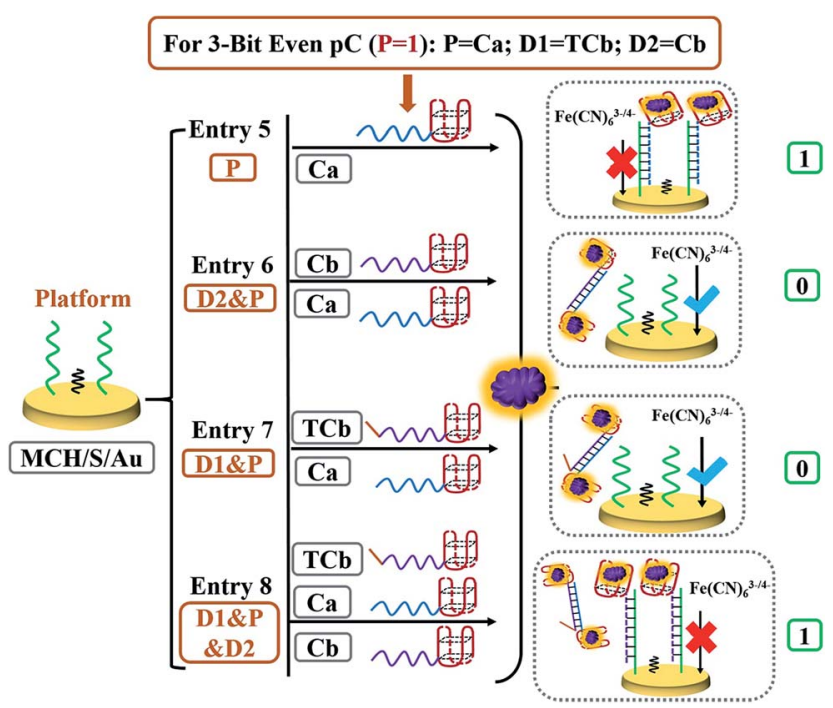

Scheme 3 Detailed DNA hybridization of different entries for the 3-bit even $\mathrm{pC}$ ( $\mathrm{P}=1$ states). Strands $\mathrm{Ca}, \mathrm{TCb}$ and $\mathrm{Cb}$ function as inputs $\mathrm{P}, \mathrm{D} 1$ and $\mathrm{D} 2$, respectively.
In the presence of input $\mathrm{P}$ (entry 5), the DNA interaction was similar to that of entry 3 (Scheme 2), yielding output $C=1$. In the coexistence of inputs D2 and P (entry 6), the operation was the same as that of entry 4 , yielding output $C=0$. For entry 7 (inputs D1 and $\mathrm{P}$ ), the pre-reaction of $\mathrm{Ca}$ and $\mathrm{TCb}$ will generate more stable duplex $\mathrm{Ca} / \mathrm{TCb}$, resulting in a slightly changed $R_{\mathrm{ct}}$ after reacting with TB (output $C=0$ ). However, in the presence of all three inputs, $\mathrm{TCb}$ (or $\mathrm{Cb}$ ) will hybridize with $\mathrm{Ca}$ and generate duplex $\mathrm{Ca} / \mathrm{TCb}$ (or $\mathrm{Ca} / \mathrm{Cb}$ ). The resident $\mathrm{Cb}$ (or $\mathrm{TCb}$ ) could still hybridize with $\mathrm{S}$ and the nanoclaw will capture TB and finally increase the $R_{\text {ct }}$ on the surface of the electrode, yielding output $C=1$ (as an example, the interaction between $\mathrm{TCb}$ and $\mathrm{Ca}$ is shown in Scheme 3). Fig. 3A presents the $\Delta R_{\mathrm{ct}}$ column bars under different input variations. The erroneous (sad face) and normal transmission (smiling face) can be easily distinguished.

\section{Cascade logic circuit and parity checker for natural numbers}

There is no doubt that a versatile system that could perform pluralistic logical functions will exhibit giant advantages. Herein, based on the universal platform ( $\mathrm{MCH} / \mathrm{S} / \mathrm{Au}$ electrode), we further constructed a cascade logic circuit [OR-2 to 1 encoder] and a parity checker for discriminating even/odd natural numbers (0-9). For the cascade logic circuit, strands $\mathrm{Cb}$ and $\mathrm{TCb}$ functioned as the input of the OR gate, whose output was the final state of the Au electrode $(\mathrm{Cb} / \mathrm{MCH} / \mathrm{S} / \mathrm{Au}$ and $\mathrm{TCb} / \mathrm{MCH} / \mathrm{S} / \mathrm{Au}$ were output " 1 ", and $\mathrm{MCH} / \mathrm{S} / \mathrm{Au}$ was output "0"). Subsequently, the output of the OR gate was applied as one input of the downstream 2 to 1 encoder, of which another input is the poly-T strand (T30) that will hardly influence the $R_{\mathrm{ct}}$. And the output definitions were just the same with the pG/pC. Fig. $4 \mathrm{~B}$ shows the $\Delta R_{\mathrm{ct}}$ column bars under various input combinations; the input-output mapping states fitted well with the corresponding truth table (Fig. 4C), suggesting the proper operation of the OR-2 to 1 encoder. Compared with simple logic gates, cascade logic circuits could perform more complicated logic computation.

Different from the $\mathrm{pG} / \mathrm{pC}$ devices that were used to detect erroneous bits through data transmission, the parity checker
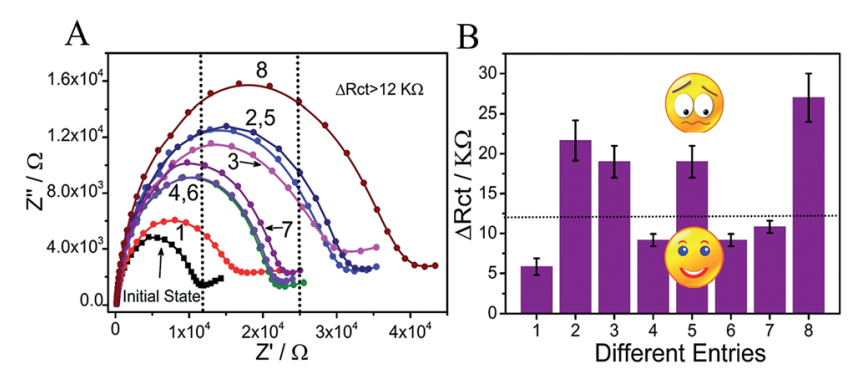

Fig. 3 (A) Electrochemical impedance spectra of the $\mathrm{MCH} / \mathrm{S} / \mathrm{Au}$ electrode under 8 different entries: entry (1) to (4) were just the same as those in Fig. 2A; (5) input $P(\mathrm{Ca})$ and TB; (6) input D2 (Cb), P (Ca) and TB; (7) input D1 (TCb), P (Ca) and TB; (8) input D1 (TCb), P (Ca) and D2 $(\mathrm{Cb})$ and $\mathrm{TB}$; $(\mathrm{B})$ the $\Delta R_{\mathrm{ct}}$ column bars of different entries. The sad and smiling faces represent erroneous and normal transmission, respectively. 


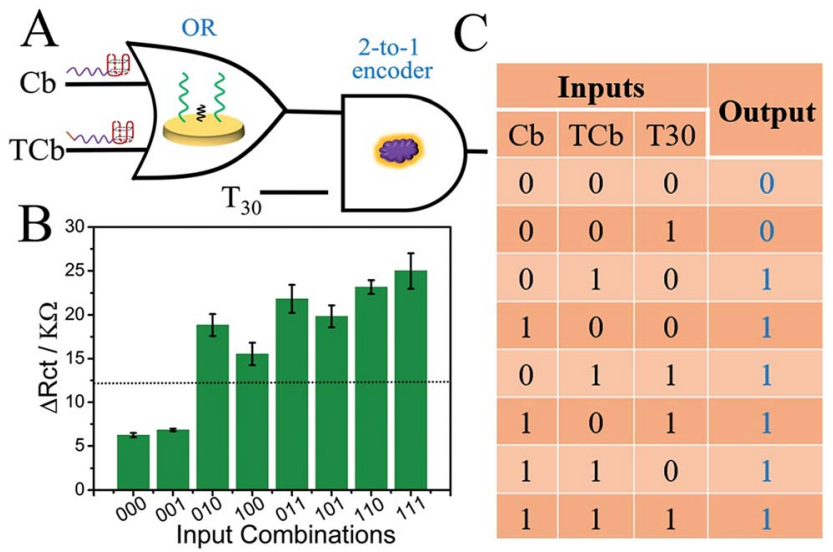

Fig. 4 (A) Equivalent logic symbol of the cascade logic circuit [OR-2 to 1 encoder]; (B) the $\Delta R_{\mathrm{ct}}$ column bars under various input variations; and $(C)$ the corresponding truth table.

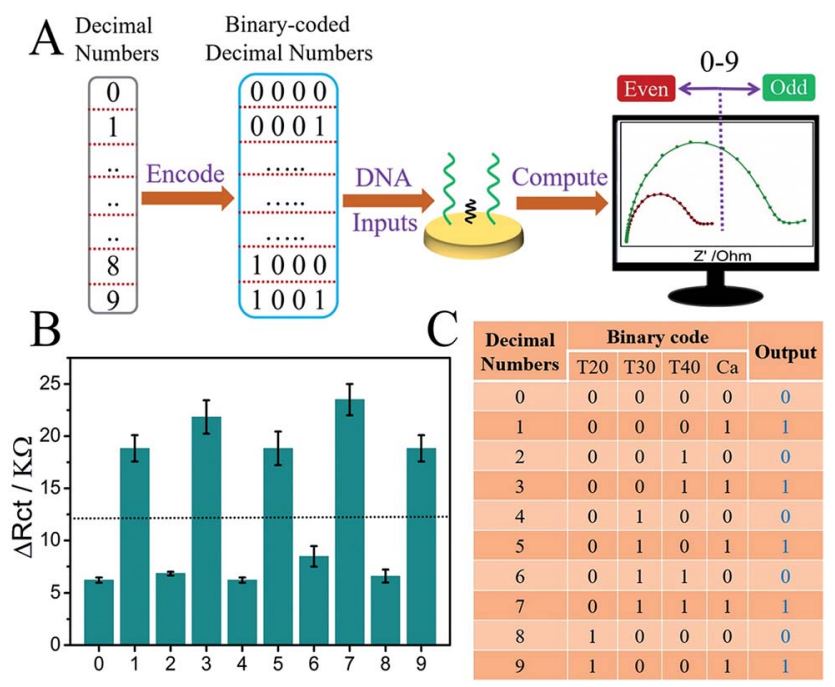

Fig. 5 (A) Schematic illustration of the parity checker for natural numbers (0-9) based on the universal system; (B) the $\Delta R_{\mathrm{ct}}$ column bars under different input combinations; and (C) the truth table.

for natural numbers could distinguish the even/odd numbers from 0 to $9{ }^{38-40}$ Here, different poly-T strands (T20, T30 and T40) and strand Ca were used as the inputs of the parity checker for natural numbers. The decimal numbers were transformed to binary-coded decimal numbers initially, and strands T20, T30, T40 and Ca functioned as the four codes, respectively. As presented in Fig. 5B, the even/odd numbers ( 0 to 9 ) can be facilely identified according to the $\Delta R_{\text {ct }}$ column bars under different input variations. All the above indicated the universal applicability of the system.

\section{Conclusion}

To summarize, by utilizing the "aptamer-nanoclaw"-modulated protein steric hindrance as label-free output and taking an even pG/pC as model device, we constructed the first electrochemical DNA pG/pC system so far. This system presented obvious advantages: firstly, this $\mathrm{pG} / \mathrm{pC}$ system was electrochemical, which largely promotes its future integration with semiconductor transistors; secondly and innovatively, a thrombin aptamer was integrated into the input-strand and acted as a "nanoclaw" to selectively capture thrombin, enabling the operation of other electrochemical DNA logic devices; thirdly, this system was simple, low cost and can be operated within $2 \mathrm{~h}$, which was comparable with previous fluorescent ones; last but not least, a cascade logic circuit and a parity checker for distinguishing even/odd natural numbers ( 0 to 9 ) were also fabricated based on the same system, which greatly met the requirements of pluralistic logical functions. This work not only opens up inspiring horizons for the design of novel electrochemical functional devices and complicated logic circuits, but also lays a solid foundation for potential logic-programmed target detection.

\section{Experimental section}

\section{Chemicals}

All the DNAs were provided by Shanghai Sangon Biotechnology (Shanghai, China); a thrombin aptamer (TBA 15) was used as a "nanoclaw" and integrated into the input strands $\mathrm{Ca}$ and $\mathrm{Cb}$. Thrombin (TB) and 6-mercaptohexanol (MCH) were purchased from Sigma Aldrich (USA). Tris (2-carboxyethyl) phosphine hydrochloride (TCEP) was purchased from Bio Basic Inc (Markham Ontario, Canada). $\mathrm{K}_{3} \mathrm{Fe}[\mathrm{CN}]_{6}$ and $\mathrm{K}_{4} \mathrm{Fe}[\mathrm{CN}]_{6}$ were obtained from Xilong Chemical Co. Ltd. (Guangdong, China). $\operatorname{NMM}(N$-methyl mesoporphyrin IX, a kind of porphyrin dye that could generate an enhanced fluorescence signal after binding with G-quadruplex) was provided by J\&K (Beijing, China). TrisHCl S-buffer (20 mM Tris, $100 \mathrm{mM} \mathrm{NaCl,} 5$ mM TCEP, pH 7.4) was used to dissolve SH-DNA. Tris-HCl M-buffer ( $20 \mathrm{mM}$ Tris, $2 \mathrm{mM} \mathrm{MCH}, \mathrm{pH}$ 7.4) was applied to block the electrode and decrease nonspecific adsorption. Tris-HCl W-buffer (5 mM Tris, $\mathrm{pH}$ 7.4) acted as the washing buffer. Other DNAs were dissolved with $1 \times$ HEPES buffer $(25 \mathrm{mM}$ HEPES, $10 \mathrm{mM} \mathrm{KCl}, 100 \mathrm{mM}$ $\mathrm{NaCl}, \mathrm{pH}$ 7.4). Distilled water was purified using a Millipore system.

\section{Apparatus}

EIS curves were collected on a Zahner Zennium electrochemical workstation (ZAHNER-Elektrik, Germany) and CV curves were measured on a CHI 760E electrochemical workstation (Shanghai Chenhua Equipments, China). A conventional three electrode electrochemical system was used, in which $\mathrm{Ag}-\mathrm{AgCl}$ acted as the reference electrode, a gold electrode (about $2 \mathrm{~mm}$ in diameter) acted as the working electrode and Pt wire functioned as the counter electrode. The CV curves $(-0.2$ to $0.6 \mathrm{~V})$ and EIS spectra $(0.1 \mathrm{~Hz}$ to $100 \mathrm{kHz})$ were collected in $5 \mathrm{mM}\left[\mathrm{Fe}(\mathrm{CN})_{6}{ }^{3-/ 4-}\right]$ and $0.1 \mathrm{M} \mathrm{KCl}$ aqueous solution. The scan rate for $\mathrm{CV}$ was $0.1 \mathrm{~V} \mathrm{~s}^{-1}$ and the oscillation potential for EIS was $5 \mathrm{mV}$.

\section{Preparation of the initial platform (MCH/S/Au electrode)}

A Au electrode was first polished with $1 \mu \mathrm{m}$ and $0.3 \mu \mathrm{m}$ alumina slurries $\left(\alpha-\mathrm{Al}_{2} \mathrm{O}_{3}\right)$ and ultrasonicated in distilled water several 
times. Then, the electrode was treated by electrochemical cleaning in $0.1 \mathrm{mM} \mathrm{H}_{2} \mathrm{SO}_{4}$, and a $\mathrm{CV}$ test was performed for about 150 cycles in the potential range of -0.3 to $1.5 \mathrm{~V}$ with a scan rate of $0.1 \mathrm{~V} \mathrm{~s}^{-1}$ to obtain the characteristic peaks of the Au electrode. After that, the electrode was washed with distilled water and dried under nitrogen gas.

Before modification, SH-DNA was dissolved in Tris-HCl Sbuffer and incubated for $1 \mathrm{~h}$ in the dark to reduce the disulfide bonds. Then, $10 \mu \mathrm{L} 1 \mu \mathrm{M}$ SH-DNA was dropped on the bare $\mathrm{Au}$ electrode and capped with a centrifuge overnight at room temperature. Then the S/Au electrode was immersed in TrisHCl M-buffer to reduce nonspecific adsorption. After washing with Tris-HCl W-buffer, the MCH/S/Au electrode was obtained.

\section{Operation of the pG/pC and other logic devices}

For the typical operation of the $\mathrm{pG} / \mathrm{pC}$, the $\mathrm{MCH} / \mathrm{S} / \mathrm{Au}$ electrode was immersed in solutions of different concentrations of $\mathrm{Ca}$ and $\mathrm{Cb}$ (for entries 2, 3, and 5) or a pre-mixture of $\mathrm{Ca} / \mathrm{Cb}$ and $\mathrm{Ca}$ / $\mathrm{TCb}$ (for entries 4, 6, 7, and 8) and reacted at room temperature for about $0.5 \mathrm{~h}$. After washing with Tris-HCl W-buffer and drying under mild nitrogen gas, $100 \mathrm{nM}$ TB was dropped on the surface of the different Au electrodes and allowed to react for about 1 h. Finally, the EIS curves of the Au electrodes were measured. The operation of the cascade logic circuit and parity checker for natural numbers was just analogous to the pG/pC, but different DNA strands were used. The concentrations of the different strands were $1 \mu \mathrm{M} \mathrm{Ca}, 0.8 \mu \mathrm{M} \mathrm{Cb}, 0.8 \mu \mathrm{M} \mathrm{TCb}$, and $200 \mathrm{nM}$ for all the poly-T strands.

\section{CD and fluorescence spectra}

CD spectra were measured from 220 to $320 \mathrm{~nm}$ in $1 \mathrm{~mm}$ pathlength cuvettes and averaged from three scans on a JASCO J820 spectropolarimeter (Tokyo, Japan). Before the fluorescence test, $500 \mathrm{nM}$ Ca and $1 \mu \mathrm{M}$ NMM were mixed in $1 \times$ HEPES buffer and incubated at room temperature for about $1 \mathrm{~h}$. Then the spectra of $\mathrm{Ca} / \mathrm{NMM}$ were collected on a Cary Eclipse Fluorescence Spectrophotometer (Agilent Technologies, USA). The spectra were collected from 550 to $750 \mathrm{~nm}$ (excitation wavelength: $399 \mathrm{~nm}$; excitation and emission slit widths: 10 and $20 \mathrm{~nm}$, respectively).

\section{Conflicts of interest}

There are no conflicts to declare.

\section{Acknowledgements}

This work was supported by the National Natural Science Foundation of China (No. 21427811 \& 21675151).

\section{Notes and references}

1 P. A. de Silva, N. H. Q. Gunaratne and C. P. McCoy, Nature, 1993, 364, 42-44.

2 J. Andreasson and U. Pischel, Chem. Soc. Rev., 2015, 44, 1053-1069.
3 E. Shapiro and B. Gil, Nat. Nanotechnol., 2007, $2,84$.

4 M. N. Stojanovic, D. Stefanovic and S. Rudchenko, Acc. Chem. Res., 2014, 47, 1845-1852.

5 J. Elbaz, O. Lioubashevski, F. Wang, F. Remacle, R. D. Levine and I. Willner, Nat. Nanotechnol., 2010, 5, 417-422.

6 I. Willner, B. Shlyahovsky, M. Zayats and B. Willner, Chem. Soc. Rev., 2008, 37, 1153-1165.

7 S. Mailloux, Y. V. Gerasimova, N. Guz, D. M. Kolpashchikov and E. Katz, Angew. Chem., Int. Ed., 2015, 54, 6562-6566.

8 A. Prokup and A. Deiters, Angew. Chem., Int. Ed., 2014, 53, 13192-13195.

9 L. Feng, Z. Lyu, A. Offenhausser and D. Mayer, Angew. Chem., Int. Ed., 2015, 54, 7693-7697.

10 L. Adleman, Science, 1994, 266, 1021-1024.

11 L. Qian and E. Winfree, Science, 2011, 332, 1196.

12 G. Seelig, D. Soloveichik, D. Y. Zhang and E. Winfree, Science, 2006, 314, 1585.

13 W. Engelen, L. H. Meijer, B. Somers, T. F. de Greef and M. Merkx, Nat. Commun., 2017, 8, 14473.

14 J. Li, A. A. Green, H. Yan and C. Fan, Nat. Chem., 2017, 9, 1056-1067.

15 X. Qu, S. Wang, Z. Ge, J. Wang, G. Yao, J. Li, X. Zuo, J. Shi, S. Song, L. Wang, L. Li, H. Pei and C. Fan, J. Am. Chem. Soc., 2017, 139, 10176-10179.

16 D. Fan, E. Wang and S. Dong, Mater. Horiz., 2017, 4, 924-931.

17 D. Fan, K. Wang, J. Zhu, Y. Xia, Y. Han, Y. Liu and E. Wang, Chem. Sci., 2015, 6, 1973-1978.

18 D. Fan, J. Zhu, Y. Liu, E. Wang and S. Dong, Nanoscale, 2016, 8, 3834-3840.

19 D. Fan, E. Wang and S. Dong, Nano Res., 2017, 10(8), 25602569.

20 D. Fan, C. Shang, W. Gu, E. Wang and S. Dong, ACS Appl. Mater. Interfaces, 2017, 9, 25870-25877.

21 H. Li, W. Hong, S. Dong, Y. Liu and E. Wang, ACS Nano, 2014, 8, 2796-2803.

22 W. Hong, Y. Du, T. Wang, J. Liu, Y. Liu, J. Wang and E. Wang, Chem.-Eur. J., 2012, 18, 14939-14942.

23 X. Yu, M. Li, T. Li, S. Zhou and H. Liu, Nanoscale, 2016, 8, 20027-20036.

24 M. Balter, S. Li, J. R. Nilsson, J. Andreasson and U. Pischel, J. Am. Chem. Soc., 2013, 135, 10230-10233.

25 S. Mandal, D. Mandal and S. K. Garai, Opt. Fiber Technol., 2014, 20, 120-129.

26 A. Kumar and S. K. Raghuwanshi, Opt. Fiber Technol., 2014, 47, 2117-2140.

27 S. Kumar, Chanderkanta and A. Amphawan, Opt. Commun., 2016, 364, 195-224.

28 D. Fan, E. Wang and S. Dong, Chem. Sci., 2017, 8, 1888-1895. 29 D. Fan, E. Wang and S. Dong, ACS Appl. Mater. Interfaces, 2017, 9, 1322-1330.

30 E. Xiong, X. Zhang, Y. Liu, J. Zhou, P. Yu, X. Li and J. Chen, Anal. Chem., 2015, 87, 7291-7296.

31 L. Wang, R. Ma, L. Jiang, L. Jia, W. Jia and H. Wang, Biosens. Bioelectron., 2017, 92, 390-395.

32 Q. Wang, Z. Zhou, Y. Zhai, L. Zhang, W. Hong, Z. Zhang and S. Dong, Talanta, 2015, 141, 247-252.

33 D. Li, S. Song and C. Fan, Acc. Chem. Res., 2010, 43, 631-641. 
34 J. Elbaz, B. Shlyahovsky, D. Li and I. Willner, ChemBioChem, 2008, 9, 232-239.

35 S. S. Mahshid, S. Camire, F. Ricci and A. Vallee-Belisle, J. Am. Chem. Soc., 2015, 137, 15596-15599.

36 D. Zhao, X. Dong, N. Jiang, D. Zhang and C. Liu, Nucleic Acids Res., 2014, 42, 11612-11621.
37 S. Chen, P. Liu, K. Su, X. Li, Z. Qin, W. Xu, J. Chen, C. Li and J. Qiu, Biosens. Bioelectron., 2018, 99, 338-345.

38 K. He, Y. Li, B. Xiang, P. Zhao, Y. Hu, Y. Huang, W. Li, Z. Nie and S. Yao, Chem. Sci., 2015, 6, 3556-3564.

39 R.-R. Gao, S. Shi, Y. Zhu, H.-L. Huang and T.-M. Yao, Chem. Sci., 2016, 7, 1853-1861.

40 M. Li, X. Yu and H. Liu, Electrochim. Acta, 2016, 220, 562-572. 